\title{
Simplified Optimum Control Method for Mono-inverter Dual Parallel PMSM Drive
}

\begin{abstract}
This paper proposes a simplified optimum control strategy for a mono-inverter dual parallel (MIDP) permanent magnet synchronous motor (PMSM). With reference to the parallel PMSM unified model, two different auxiliary conditions are investigated. The first minimizes the supplied inverter current, and the second maximizes the motor's efficiency. Their formal expressions are derived by the introduction of three new normalized variables, linked to the load conditions. Thus, the optimum tracking considerations are general, and the parameters are independent. Simplified closed analytical forms are found for the optimum tracking, independently under steady state and transient conditions. The selection of the best solution is then found via a parameter independent look-up table, and imposed through a closed loop control diagram. The proposed control is compared to the present state of the art via experiments on a $1400 \mathrm{~W}$ MIDP PMSM test bench, showing an equivalent performance, with a lower computational burden.
\end{abstract}

Index Terms-maximum torque per ampere, monoinverter dual parallel motor drive, permanent magnet motor, simplified control strategy

\section{NOMENClAtuRE}

$R \quad$ Stator resistance

$L \quad$ Synchronous inductance

$\Phi_{r} \quad$ Rotor flux linkage

$p \quad$ Pole pairs

$\omega \quad$ Electrical angular speed

$\theta \quad$ Rotor position

$\mathbf{v}, v_{d}, v_{q}$ Voltage space vector and d-q components

$\mathbf{i}, i_{d}, i_{q} \quad$ Current space vector and d-q components

$T \quad$ Electromagnetic torque

Attribute/Superscript/Subscript

* Reference; $\Sigma$ Semi-sum; $\Delta$ Semi-difference

\section{INTRODUCTION}

$\mathrm{T}$ HE major progress in power electronics that began in the 70s led to a significant reduction in the cost and large improvement in the performance of electrical drives. For some applications, the cost is the strictest constraint, whereas for others, the performance is the most important characteristic for an electrical drive. For this reason, different solutions for electrical drives have been studied and developed in recent years. In this scenario, several solutions using only one inverter to drive two motors connected in parallel have been proposed. In the technical literature, these solutions are called mono-inverter dual parallel (MIDP) motors. MIDP machines have spread to applications where improving the performance is not the main goal but cost reduction is important. It is worth noting that, since MIDP solutions are intended for low power applications, where the use of integrated ac/ac converters is preferred, it could make possible to remove not only the last VSI but also the inherent duplicates, i.e., the filter, rectifier, controller, and protection circuit (see Fig. 1).

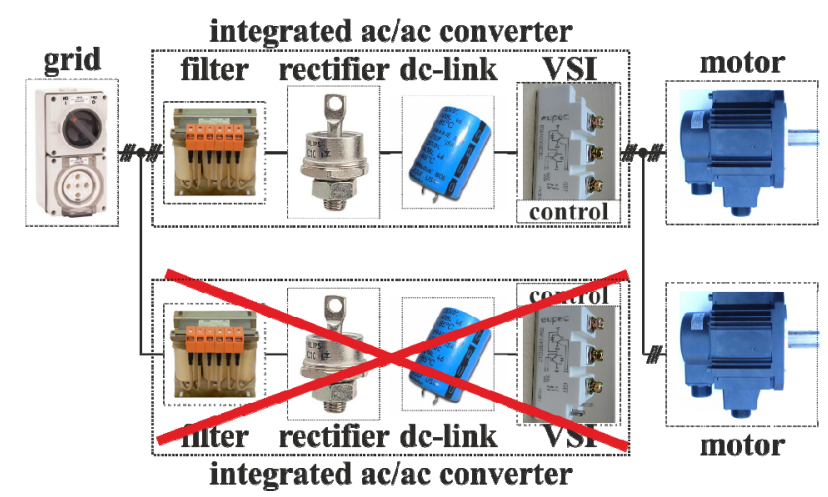

Fig. 1. Mono-inverter Dual Parallel Motor Drive System

In recent years, several solutions employing MIDP machines have been proposed for applications where high dynamic performances are not required, i.e., fan coils, railway traction, and conveyor belts [1].

The most commonly used machine for MIDP drives is the induction motor (IM). Indeed, two IMs supplied with the same frequency can operate at different speeds via a differential slip frequency; moreover, the intrinsic damping factor of the squirrel cage improves the dynamic stability of the two motors by reducing speed oscillations [2]-[6]. In any case, in several papers, MIDP-equipped permanent magnet synchronous machines (PMSMs) have been proposed because of their higher torque and power density in comparison with IMs [1], [7]-[21].

The first authors presenting MIDP PMSMs were Bidarti et al. [7]-[12]. They proposed a method to control only one of the two motors in a master-slave approach, by setting to zero the direct reference current of the controlled motor, usually the one supplying the highest power. However, the resulting working point does not correspond to any optimal operating condition, and thus neither the efficiency nor performances are optimized. Moreover, pendulum oscillations between the two 
motors occur and are not damped by the control.

In [13]-[15], Spina et al. introduced a unified model to control a virtual machine behaving like an average machine. The unified model was used to determine the optimal phase displacement of the two rotors, which made it possible to minimize the inverter currents. However, the feed forward proposed control scheme does not guarantee that the optimal condition is reached; furthermore, since the optimal operating condition is tracked by using a simple first order solution, the system performance is not always maximized. These are the main differences with respect to this work, together with a generalized formulation of the optimization problem.

In [16], Lazi et al. proposed a unified model that was very similar to that of [13] and configured the control strategy by imposing a zero value for the direct current of the virtual machine. This auxiliary condition was neither an optimum for the efficiency nor for the performances.

Lee-Ha et al., in [1], [17]-[22], solved the model for the two machines separately without using the unified model. Only one motor was controlled in a master-slave approach, and the proposed auxiliary condition was a reference value for the direct current of the master machine, which optimized the efficiency of the overall system. The optimal condition can be found by solving a $4^{\text {th }}$ order algebraic equation, which gives a viable operating point only at the steady state. Since the control reference linked to this optimal condition would lead to strong oscillations in transient operations, a squirrel-cage like damping term is added to the actual control reference.

In this paper, the unified model of the virtual average machine is used, and new variables are introduced such that the considerations about the optimum tracking are independent on the motor parameters. Two different auxiliary conditions are considered: the first minimizing the inverter supplied current, and the second maximizing the motors' efficiency. The associated computational problem is solved by introducing a parameter independent look-up table, which makes it possible to switch between the two simplified solutions. In this way, a closed analytical form is found for all the controlled variables, regardless of the actual system condition (steady state or transient). A complete closed loop control diagram is presented, containing a detailed explanation for the current limitation procedure. The parameter uncertainty problem is also investigated. In order to highlight the innovative and good content of the proposed control strategy, some experimental tests were carried out comparing the performance of the proposed method with that of [1], which can be considered the present state of the art with respect to MIDP PMSM drives. The comparison highlights that the proposed simplified control strategy shows equivalent performance, although at a lower computational burden.

The paper is structured as follows. In section III, the mathematical model is illustrated; in section IV the control strategy is analyzed. The control diagram is proposed in section $\mathrm{V}$, while the parameter variation analysis is reported in section VI. Finally, the experimental results are presented in section VII.

\section{MATHEMATICAL MODEL}

A synthetic mathematical model for two identical, parallelconnected PMSMs (motor A and motor B) can be derived from the well-known model written for a single machine in its own synchronous frame:

$$
\left\{\begin{array}{l}
\mathbf{v}_{K}=R \mathbf{i}_{K}+L \frac{d \mathbf{i}_{K}}{d t}+j \omega_{K} L \cdot \mathbf{i}_{K}+j \omega_{K} \Phi_{r} \\
T_{K}-T_{r, K}=\frac{J}{p} \frac{d \omega_{K}}{d t} ; \quad T_{K}=\frac{3}{2} p \Phi_{r} \operatorname{Im}\left\{\mathbf{i}_{K}\right\} \\
\omega_{K}=\frac{d \theta_{K}}{d t}
\end{array}\right.
$$

where $K \in\{A, B\}$.

By defining the common mode and differential quantities: $G_{\Sigma}=\left(G_{A}+G_{B}\right) / 2 ; G_{\Delta}=\left(G_{A}-G_{B}\right) / 2$

model (1) can be rewritten with respect to the "mean reference frame" $d, q$ of Fig. 2 whose instantaneous position corresponds to the angle $\theta_{\Sigma}$.

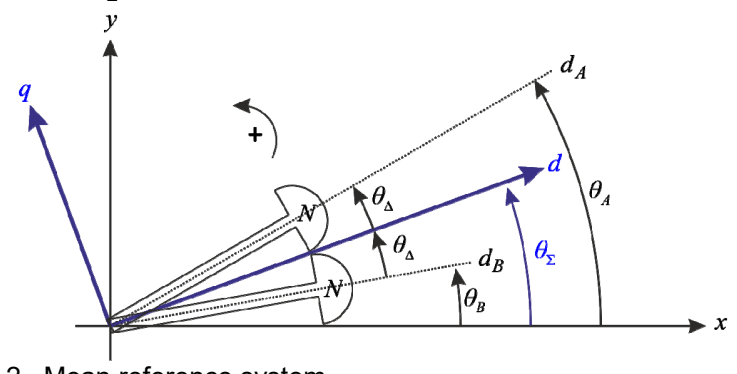

Fig. 2. Mean reference system

Thus, model (1) becomes:

$$
\left\{\begin{array}{l}
\mathbf{v}_{\Sigma}=R \mathbf{i}_{\Sigma}+L \frac{d \mathbf{i}_{\Sigma}}{d t}+j \omega_{\Sigma} L \mathbf{i}_{\Sigma}+j \omega_{\Sigma} \Phi_{r} \cos \theta_{\Delta}-\omega_{\Delta} \Phi_{r} \sin \theta_{\Delta} \\
T_{\Sigma}-T_{r, \Sigma}=\frac{J}{p} \frac{d \omega_{\Sigma}}{d t} ; T_{\Sigma}=\frac{3}{2} p \Phi_{r} \operatorname{Im}\left\{\mathbf{i}_{\Sigma} \cos \theta_{\Delta}-\mathbf{i}_{\Delta} j \sin \theta_{\Delta}\right\} \\
\omega_{\Sigma}=\frac{d \theta_{\Sigma}}{d t} \\
0=R \mathbf{i}_{\Delta}+L \frac{d \mathbf{i}_{\Delta}}{d t}+j \omega_{\Sigma} L \mathbf{i}_{\Delta}-\omega_{\Sigma} \Phi_{r} \sin \theta_{\Delta}+j \omega_{\Delta} \Phi_{r} \cos \theta_{\Delta} \\
T_{\Delta}-T_{r, \Delta}=\frac{J}{p} \frac{d \omega_{\Delta}}{d t} ; T_{\Delta}=\frac{3}{2} p \Phi_{r} \operatorname{Im}\left\{\mathbf{i}_{\Delta} \cos \theta_{\Delta}-\mathbf{i}_{\Sigma} j \sin \theta_{\Delta}\right\} \\
\omega_{\Delta}=\frac{d \theta_{\Delta}}{d t}
\end{array}\right.
$$

The chosen approach reduces the two motors to a single equivalent motor $\Sigma$ driven by $\mathbf{v}_{\Sigma}$ and influenced by the load imbalance through the state variables of the equivalent differential system $\Delta$. It is worth noting that, while the mean current $\mathbf{i}_{\Sigma}$ can be driven via the converter output voltages, the recirculating current $\mathbf{i}_{\Delta}$ is constrained only by the state variables.

\section{CONTROL STRATEGY}

Under steady state conditions, the model (3) becomes 


$$
\left\{\begin{array}{l}
\mathbf{v}_{\Sigma}=R \mathbf{i}_{\Sigma}+j \omega_{\Sigma} L \mathbf{i}_{\Sigma}+j \omega_{\Sigma} \Phi_{r} \cos \psi \\
T_{r, \Sigma}=\frac{3}{2} p \Phi_{r} \operatorname{Im}\left\{\mathbf{i}_{\Sigma} \cos \psi+\mathbf{i}_{\Delta} j \sin \psi\right\} \\
0=R \mathbf{i}_{\Delta}+j \omega_{\Sigma} L \mathbf{i}_{\Delta}+\omega_{\Sigma} \Phi_{r} \sin \psi \\
T_{r, \Delta}=\frac{3}{2} p \Phi_{r} \operatorname{Im}\left\{\mathbf{i}_{\Sigma} j \sin \psi+\mathbf{i}_{\Delta} \cos \psi\right\}
\end{array}\right.
$$

where $\psi=-\theta_{\Delta}$.

Once the mechanical properties of the system have been assigned by fixing $T_{r, \Sigma}, T_{r, \Delta}, \omega_{\Sigma}$ values, the six scalar equations (two scalar and two vectorial) defined by the model (4) constrain the steady state values of the variables $\mathbf{v}_{\Sigma}, \mathbf{i}_{\Sigma}, \mathbf{i}_{\Delta}, \psi$. The resulting degree of freedom can be exploited by defining an appropriate control strategy. With reference to the equivalent motor $\Sigma$, the typical maximum torque per ampere (MTPA) condition is obtained by maximizing the ratio $\mathrm{P}_{C}=T_{\Sigma} /\left|\mathbf{i}_{\Sigma}\right|$, which also leads to minimizing the currents supplied by the converter (as in [13]-[15]). Alternatively, a different strategy could aim to minimize the motor currents (as in [1]) by maximizing the ratio $\mathrm{P}_{M}=\sqrt{2} T_{\Sigma} / \sqrt{\left(\left|\mathbf{i}_{A}\right|^{2}+\left|\mathbf{i}_{B}\right|^{2}\right)}$. By defining the maximum torque/current ratio $\mathrm{P}_{\max }=1.5 p \Phi_{r}$ obtainable when a single motor is fed by a single converter, it is easy to verify that the normalized ratios $\rho_{M}=\mathrm{P}_{M} / \mathrm{P}_{\text {max }}$ and $\rho_{C}=\mathrm{P}_{C} / \mathrm{P}_{\max }$ can be synthetically expressed as follows:

$\rho=\left|\xi_{\Sigma} \sin (2 \psi)\right| \cdot\left[\left(2 \xi_{\Sigma} \xi_{\Delta} \cos \psi-\xi_{\omega}^{2} \sin (2 \psi) \cos \psi\right)^{2}+\right.$

$$
\left.+\left(2 \xi_{\Sigma} \sin \psi+2 \xi_{\omega} \sqrt{1-\xi_{\omega}^{2}} \sin ^{3} \psi\right)^{2}+M\left(\xi_{\omega} \sin (2 \psi) \sin \psi\right)^{2}\right]^{-\frac{1}{2}}
$$

where $M=1$ for $\rho_{M}, M=0$ for $\rho_{C}$, and

$\xi_{\Delta}=\frac{T_{\Delta}}{T_{\Sigma}} ; \quad \xi_{\Sigma}=\frac{T_{\Sigma}}{T_{s}} ; \quad \xi_{\omega}=\frac{p \omega_{r} L}{Z} ;$

$T_{s}=\frac{3 p}{2} \frac{\Phi_{r}^{2}}{L} ; \quad Z=\sqrt{R^{2}+\left(\omega_{\Sigma} L\right)^{2}}$

Differently from [13]-[15], the choice of the coefficients $\xi_{\Sigma}, \xi_{\Delta}, \xi_{\omega}$ makes the expression of $\rho_{M}$ and $\rho_{C}$ formally independent of the motor parameters and highlights that the maximum obtainable values for the two ratios depend on the load conditions $\left(T_{\Sigma}, T_{\Delta}, \omega\right)$. For any assigned load condition, it is possible to calculate two different optimum values for $\psi$ corresponding to maximum values of $\rho_{M}$ and $\rho_{C}$. Because the ratios $\rho_{M}, \rho_{C}$ are high-order nonlinear transcendent functions, it is not possible to solve the optimum problem in a closed form, unless the trigonometric functions are developed in a Taylor series and an approximation is introduced. Although $\rho_{M}$ and $\rho_{C}$ are related to two different control strategies, the first making it possible to obtain the minimum motor losses, and the second the minimum converter size, the $1^{\text {st }}$ and $2^{\text {nd }}$ order approximated optimum solutions are the same for both ratios and correspond to the following: $\psi_{o p t}^{(1)}=\frac{\xi_{\Delta} \xi_{\Sigma}}{\xi_{\omega}^{2}} \quad ; \quad \psi_{o p t}^{(2)}=\frac{-3 \xi_{\omega}^{2}+\sqrt{9 \xi_{\omega}^{4}+96 \xi_{\Delta}^{2} \xi_{\Sigma}^{2}}}{16 \xi_{\Delta} \xi_{\Sigma}}$

As an example, in Fig. 3, the optimum $\rho_{M}$ value is depicted together with the ones resulting from the $1^{\text {st }}$ and $2^{\text {nd }}$ order approximations for different load conditions and with respect to the load imbalance $\xi_{\Delta}$.

As can be noticed in Fig. 3. $a, b$, the $1^{\text {st }}$ order solution gives a better performance for high $\xi_{\omega}$, while it shows a significant distance from the optimum at low speed $(c, d)$; indeed, $\psi_{o p t}^{(1)}$ presents a singularity at zero speed (see (6)). On the other hand, the behavior linked to the $2^{\text {nd }}$ order solution appears to be more robust and remains closer to the optimum at low speed $(\mathrm{c}, d)$; a similar behavior and considerations could be shown for $\rho_{C}$.
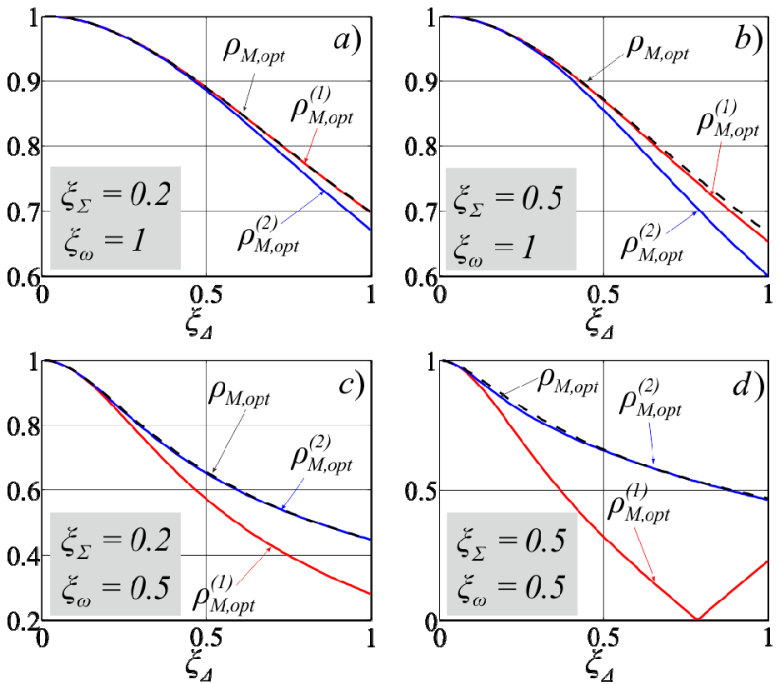

Fig. 3. Optimum $\rho_{M}$ and approximated 1st and 2nd order optimum values for different load conditions

In order to simplify the optimization strategy exposition, the following considerations will be made only with reference to $\rho_{M}$ and therefore from now on the notation $\rho \equiv \rho_{M}$ will be used.

Hence, differently from [13]-[15], which does not involve the $2^{\text {nd }}$ order approximation, in the present work a criterion is formulated for dynamically selecting the best approximation order solution; in particular, each pair of $\xi_{\omega}, \xi_{\Delta}$ identifies the set $\xi_{\Sigma, 12}$ (hence denoted as the 2-D switching boundary), where the $1^{\text {st }}$ and $2^{\text {nd }}$ order solutions determine the same $\rho_{M}$; the correspondent ratios satisfy the following:

$\rho_{\text {opt }}^{(1)}>\rho_{\text {opt }}^{(2)} \quad \forall \xi_{\Sigma}<\xi_{\Sigma, 12} \quad, \quad \rho_{\text {opt }}^{(2)}>\rho_{\text {opt }}^{(1)} \forall \xi_{\Sigma}>\xi_{\Sigma, 12}$

As can be deduced from Fig. 4, $\xi_{\Sigma, 12}\left(\xi_{\omega}, \xi_{\Delta}\right)$ depends mostly on $\xi_{\omega}$, while its variation with respect to $\xi_{\Delta}$ is weaker. This behavior, which is independent of the motor parameters, suggests that just one curve $\xi_{\Sigma, 12}\left(\xi_{\omega}, \bar{\xi}_{\Delta}\right)$ can be used as the approximation switching criterion for the optimal angle evaluation: 


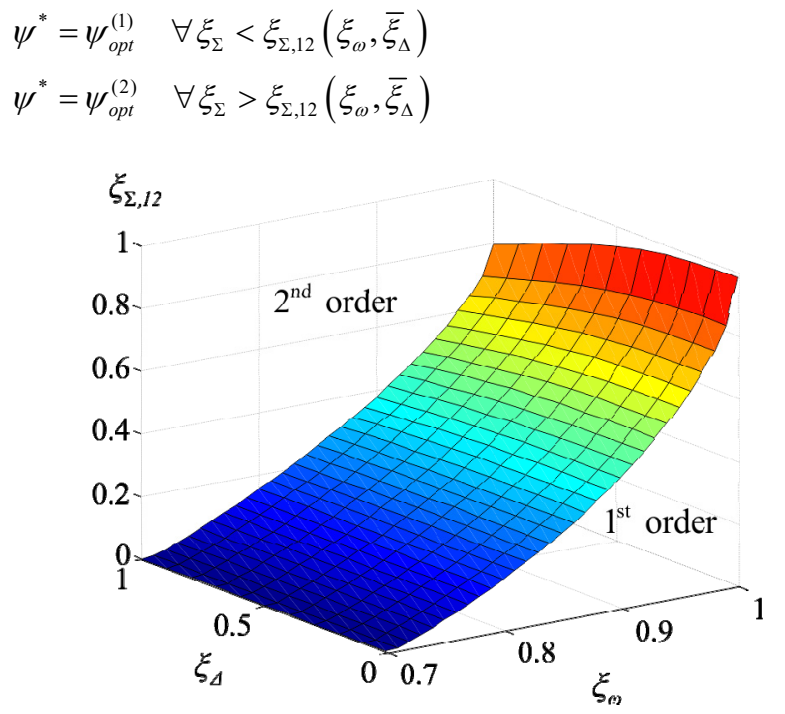

Fig. 4. 2-D switching boundary

An appropriate choice of $\bar{\xi}_{\Delta}$ value should minimize the maximum possible value of the relative error $\varepsilon_{12}=\left|\rho_{\text {opt }}^{(1)}-\rho_{\text {opt }}^{(2)}\right| / \max \left(\rho_{\text {opt }}^{(1)}, \rho_{\text {opt }}^{(2)}\right)$. Fig. $5 . a$ depicts the maximum error $\varepsilon_{12}$ that can occur in the range $\xi_{\Delta}=[0,1]$ as a function of $\bar{\xi}_{\Delta}$ and for different values of $\xi_{\omega}$. As can be noticed, regardless of the considered $\xi_{\omega}$ value, the minimum is located around $\bar{\xi}_{\Delta}=0.91$.

The 1-D switching boundary $\xi_{\Sigma, 12}\left(\xi_{\omega}, 0.91\right)$ leads to the $\varepsilon_{12}$ behavior depicted in Fig. 5.b, which confirms that $\varepsilon_{12}<1.5 \%$ under all operating conditions.
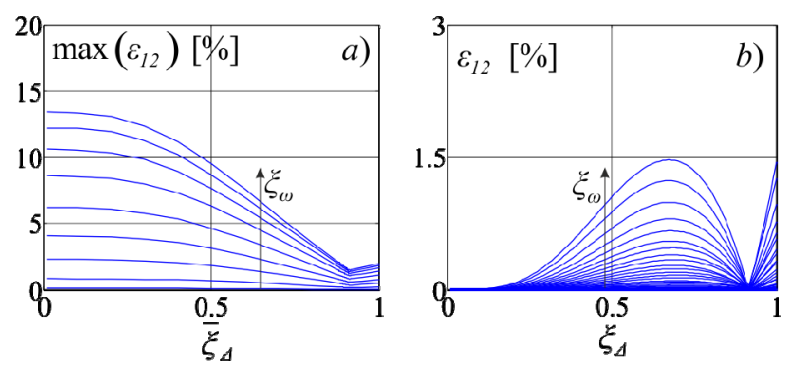

Fig. 5. a): Maximum error $\varepsilon_{12}$ that can occur in the range $\xi_{\Delta}=[0,1]$;

b) : Error $\varepsilon_{12}$ for the chosen switching boundary $\xi_{\Sigma, 12}\left(\xi_{\omega}, 0.91\right)$.

Finally, Fig. 6 shows the maximum relative error $\varepsilon=\left|\rho_{\text {opt }}-\rho_{\text {opt }}^{*}\right| / \rho_{\text {opt }}$ that can occur when the optimum angle $\psi^{*}$ is chosen as per (8).

Analogous considerations could be made for $\rho_{C}$, leading to similar behaviors.

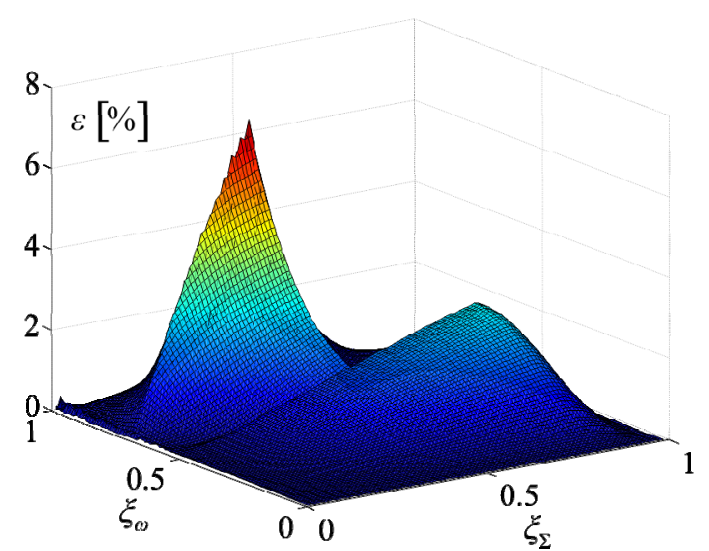

Fig. 6. Maximum error $\varepsilon$ as per (8) with $\xi_{\Sigma, 12}\left(\xi_{\omega}, 0.91\right)$.

\section{CONTROL DIAGRAM}

The proposed control diagram for the MIDP PMSM drive system is schematically represented in Fig. 7.

The previously discussed control strategy is applied as per (8) by means of the 1-D pre-built look-up table $\xi_{\Sigma, 12}\left(\xi_{\omega}, 0.91\right)$. The calculated $\psi^{*}$ is compared with the actual semi-displacement of the rotor positions $\psi$ of the two motors, and the error is processed using a $\mathrm{P}$ regulator, giving the reference value of the $\Delta$ rotational speed $\left(\omega_{r \Delta}^{*}\right)$; naturally, in the context of digital control, a delay of one sampling time is applied. Differently from [13]-[15], the closed loop control of $\psi$, guarantees that the optimal condition is actually reached.

The reference speed values in terms of $\Sigma$ and $\Delta$ are separately processed using two PI regulators, giving the corresponding torque reference values $T_{\Sigma}^{*}, T_{\Delta}^{*}$. The not-limited reference values of the $\Sigma$ current components can be easily derived using the last three of (4), giving the following expressions:

$\left\{\begin{array}{l}i_{d \Sigma, n l}^{*}=\frac{2 T_{\Delta}^{*}}{3 p \Phi_{r}} \frac{1}{\sin \psi}-\frac{\omega_{\Sigma}^{2} \Phi_{r} L}{R^{2}+\omega_{\Sigma}^{2} L^{2}} \cos \psi \\ i_{q \Sigma, n l}^{*}=\frac{2 T_{\Sigma}^{*}}{3 p \Phi_{r}} \frac{1}{\cos \psi}+\frac{\omega_{\Sigma} \Phi_{r} R}{R^{2}+\omega_{\Sigma}^{2} L^{2}} \frac{\sin ^{2} \psi}{\cos \psi}\end{array}\right.$

As in [1], the singularity linked to the $1 / \sin \psi$ terms of (9) is linearized around zero in order to avoid abrupt changes in the $d$ reference current component. 


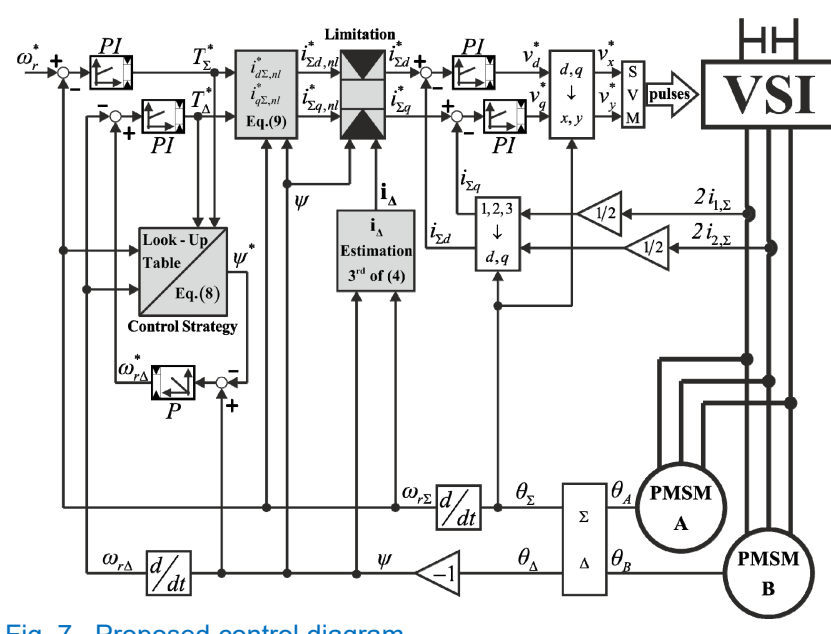

Fig. 7. Proposed control diagram.

In order to ensure that the magnitude of the currents flowing in motors $\mathrm{A}$ and $\mathrm{B}$ do not overcome the limit value $I_{L}$ and that the direct components do not reach the demagnetizing limit value $I_{s}$, a limitation procedure is applied as schematically represented in Fig. 8 (see Appendix), where $\{L\}$ and $\{S\}$ are the acceptable ranges with respect to the magnitude and demagnetizing current constraints, respectively.

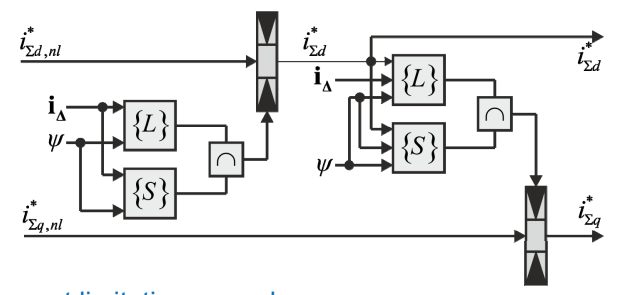

Fig. 8. Current limitation procedure.

The proposed limitation procedure gives priority to the differential torque by first computing the admissible $i_{\Sigma d}$ range at $i_{\Sigma q}=0$. Then, the admissible range of the $i_{\Sigma q}$ current is dynamically determined based on the actual limited $i_{d \Sigma}^{*}$ reference value.

Once the reference current components $i_{d \Sigma}^{*}, i_{q \Sigma}^{*}$ are available, the reference voltage components in the rotating mean reference frame $v_{d}^{*}, v_{q}^{*}$ are obtained by means of a double PI regulator and a traditional decoupling control. Then, they are transformed into static reference frame components $v_{x}^{*}, v_{y}^{*}$ via angle $\theta_{\Sigma}$; these voltages are applied using a classical SVM technique.

In the proposed control diagram (Fig. 7), only the two converter phase currents are measured, while the $\Delta$ current is estimated via the third of (4), since it is induced by the term $\omega_{\Sigma} \Phi_{r} \sin \psi$ and therefore is characterized by a slow dynamic.

Considering that low power motors are often equipped with internal low cost resolver, which do not significantly affect the total cost, Fig. 7 refers to a sensored context. Anyway, in the case where the two motors are not provided with position sensors, the proposed control can always operate in tandem with a position estimation technique (as in [1]). Naturally, in the context of sensorless motors, four current sensors would be necessary; it is worth noting that the control of [1] always needs four current sensors, regardless of sensored or sensorless context.

\section{PARAMETER VARIATION SENSITIVITY}

In the context of a real MIDP PMSM drive, the parameters of the two motors may differ as a result of the manufacturing tolerance. Moreover, significant differences in the motor parameters can arise as a result of uneven magnetic saturation and/or winding temperature conditions, as a consequence of load imbalances.

In order to analyze the robustness of the proposed control in relation to the parameter uncertainties, the relative current increment $\chi=\sqrt{\left(\tilde{I}_{A}^{2}+\tilde{I}_{B}^{2}\right) /\left(I_{A}^{2}+I_{B}^{2}\right)}-1$ (hence defined as the control parameters sensitivity) is considered, with $\tilde{I} / I$ the currents RMS in presence/absence of parameter variations. The values of $\chi$ are reported in Fig. 9 in the presence of the parameter variations $\quad\left|\Delta R_{\Sigma}\right|+\left|\Delta R_{\Delta}\right| \leq 10 \%$, $\left|\Delta L_{\Sigma}\right|+\left|\Delta L_{\Delta}\right| \leq 10 \%$, and $\left|\Delta \Phi_{r \Sigma}\right|+\left|\Delta \Phi_{r \Delta}\right| \leq 10 \%$ at the rated speed and $\xi_{\Sigma}=0.5$; the quantities $\Delta_{\Sigma}, \Delta_{\Delta}$ are defined as per (2), i.e. they represent the relative common mode, differential mode errors with respect to the motor parameters. In Fig. 9, the proposed control algorithm parameter sensitivity (black continuous line) is compared with the one of the method [1] (blue dashed line); the two methods are denoted as Proposed Control (PC) and Master Slave Control (MSC).
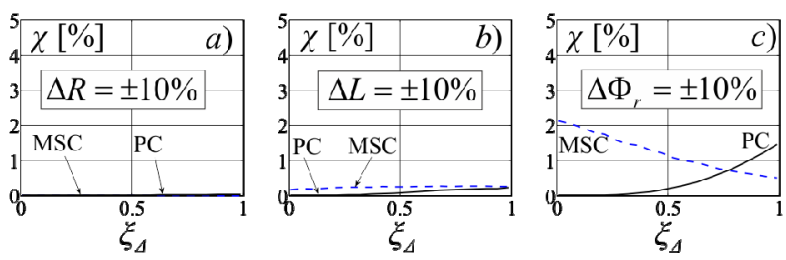

Fig. 9. Relative current increment $\chi$ due to parameter deviation for PC (black line) and MSC (blue dashed line) at rated speed and $\xi_{\Sigma}=0.5$ in presence of $\pm 10 \%$ parameter variation for a) stator resistance, b) synchronous inductance, and c) rotor flux linkage.

As can be noted, the quantity $\chi$ is less sensitive to $\Delta R$ and more sensitive to $\Delta \Phi_{r}$. On the other hand, the rotor flux linkage can be estimated with high accuracy (using a no load test) and is expected to vary much less than the stator resistance, which has a wider range of variation as a result of the stronger temperature dependence. Anyway, Fig. 9 testifies that MIDP systems are highly insensitive to parameter errors and therefore the optimum point is always tracked with good accuracy; similar results were found for different $\xi_{\Sigma}$ and $\xi_{\omega}$ values.

\section{EXPERIMENTAL RESULTS}

In order to prove the effectiveness of the proposed control (PC), after extended numerical analyses, the algorithm was experimentally tested and compared to the method reported in [1] (MSC). 


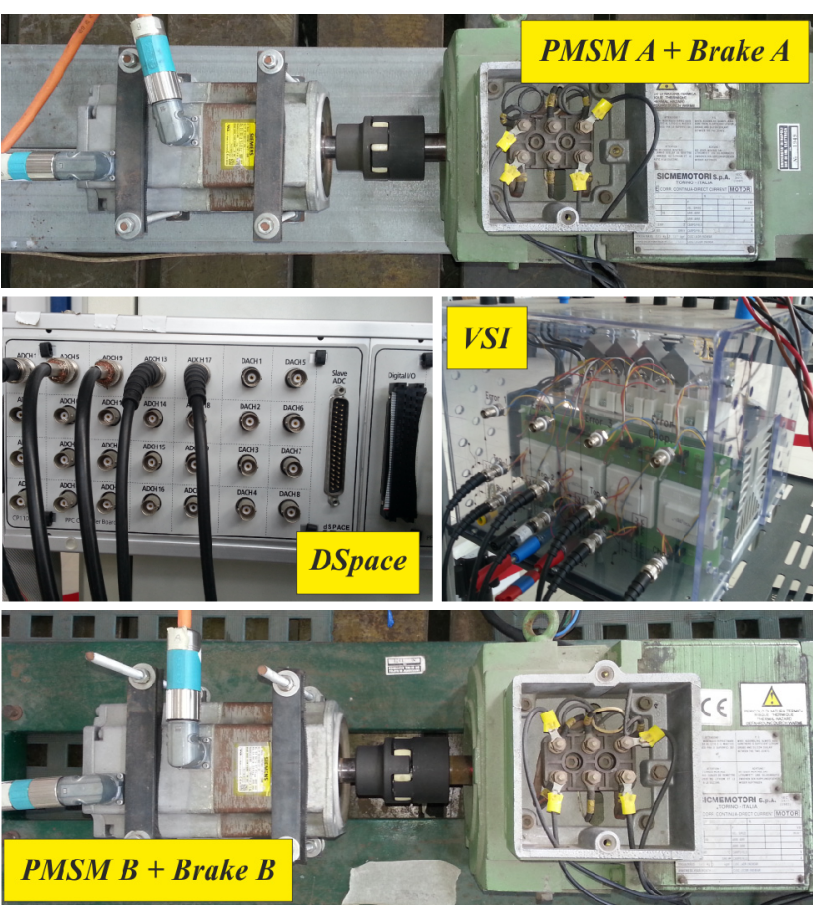

Fig. 10. Experimental laboratory prototype.

The test bench was composed of a DSpace platform Controller Board DS1103, a 3-phase converter (market name: SEMITEACH B6U+E1CIF+B6CI) using Semikron IGBT SKM50GB123D,

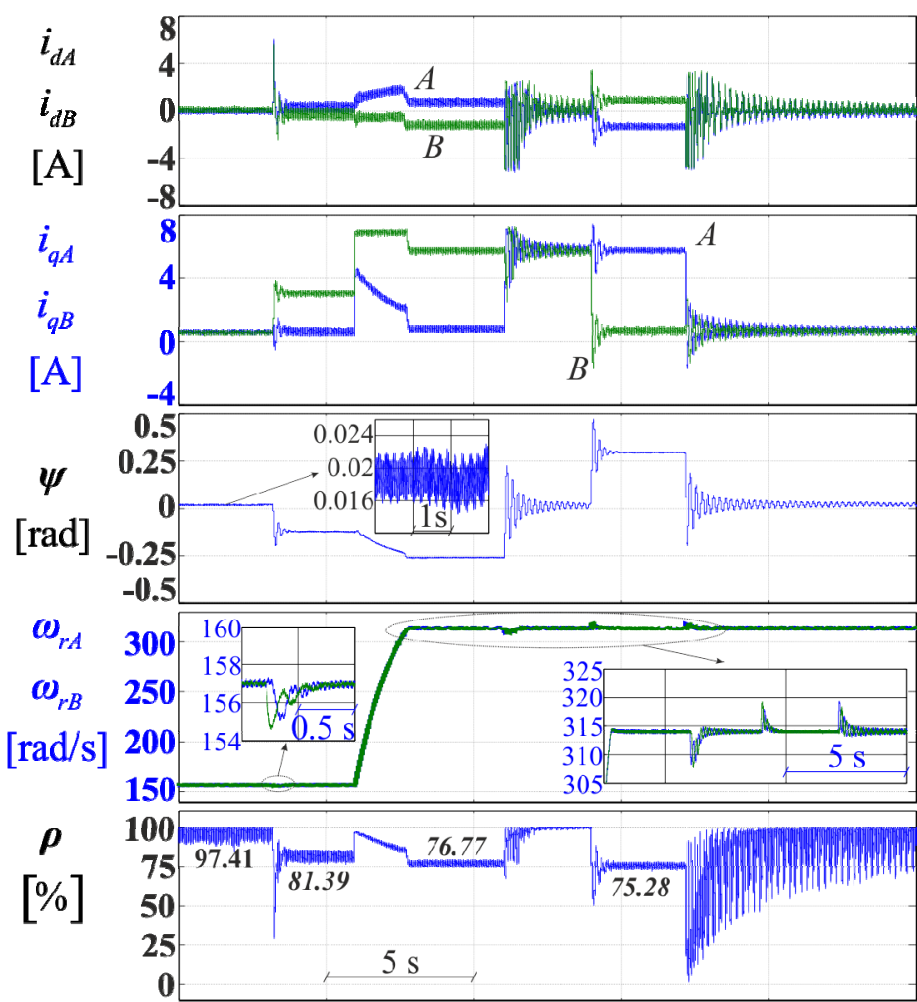

(a)
TABLE I

MAIN DATA OF EXPERIMENTAL TEST BENCH

\begin{tabular}{cll} 
Symbol & \multicolumn{1}{c}{ Quantity } & \multicolumn{1}{c}{ Value } \\
\hline$R$ & Stator resistance & $0.74 \Omega$ \\
$L$ & Synchronous inductance & $20 \mathrm{mH}$ \\
$\Phi_{r}$ & Rotor flux linkage & $0.1738 \mathrm{~Wb}$ \\
$p$ & Pole pair & 3 \\
- & Rated power & $1.4 \mathrm{~kW}$ \\
- & Rated torque & $4.4 \mathrm{Nm}$ \\
- & Rated speed & $314 \mathrm{rad} / \mathrm{s}$ \\
- & Rated voltage (rms) & $240 \mathrm{~V}$ \\
- & Rated current (rms) & $4 \mathrm{~A}$ \\
- & Total inertia & $0.0230 \mathrm{Kg} \mathrm{m}$ \\
- & Switching frequency & $5 \mathrm{kHz}$ \\
- & Currents regulators bandwidth & $160 \mathrm{~Hz}$ \\
- & $\Delta$ speed regulator bandwidth & $10 \mathrm{~Hz}$ \\
- & $\psi$ regulator bandwidth & $9 \mathrm{~Hz}$ \\
- & $\sum$ speed regulator bandwidth & $5 \mathrm{~Hz}$ \\
\hline \hline
\end{tabular}

two Siemens PMSM motors (1FK7044-7AF71 series) equipped with an internal low cost resolvers, two identical separately excited DC generators used as brakes by means of load resistors, and all of the necessary LEM hall effect transducers; the main data of the prototype shown in Fig. 10 are summarized in Table I.

In order to achieve a fair comparison between the two approaches, the regulators of both control schemes were set with equal constants.

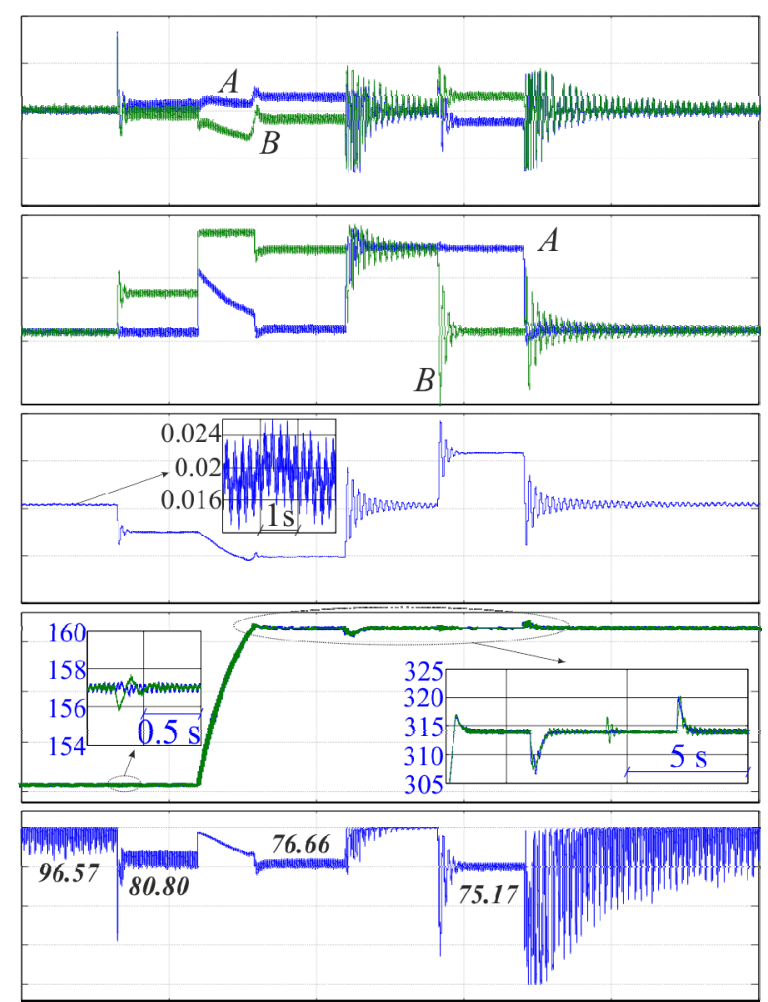

(b)

Fig. 11. Experimental results during load sequence and speed reference change showing $i_{d A}, i_{d B}, i_{q A}, i_{q A}, \psi, \omega_{r A}, \omega_{r B}, \rho:$ a) PC; b) MSC. 
Moreover, the proportional constant of the $\omega_{r \Delta}$ PI regulator was set to the damping gain value of the MSC. A value of 0.05 rad was chosen for the width of the $\csc \psi$ (cosecant of $\psi$ ) linearization for both controls (see (9)). Finally, in order to limit the currents during transients, the same current limitation procedure used for the PC was added to the MSC algorithm.

Starting with unloaded motors at an angular speed of $0.5 \mathrm{pu}$, Fig. 11 proposes the time behaviors of the main variables ( $a$ : PC; $b$ : MSC) during the following events:

- around $t=3 \mathrm{~s}$, motor B (slave motor for MSC) is loaded at the rated value,

- around $t=6 \mathrm{~s}$, the reference speed is stepped up to $1 \mathrm{pu}$,

- around $t=11 \mathrm{~s}$, motor $\mathrm{A}$ is loaded at the rated value,

- around $t=14 \mathrm{~s}$, motor B is unloaded,

- around $t=17 \mathrm{~s}$, motor A is unloaded.

As can be noted, despite the fact that the MSC and PC use substantially different approaches, the results are quite similar in terms of the settling times, over/under shoots, and steady state values. Nevertheless, the differences on the dynamic behaviors regarding the two control methods can be pointed out considering that while the MSC only drives the motor A, the PC drives the mean motor.

Indeed, about the MSC (column b), there are no appreciable overshoots on $i_{q A}$ current behavior (blue line) in correspondence of the load contingencies; this is particularly evident at $t=3 \mathrm{~s}, 14 \mathrm{~s}, 17 \mathrm{~s}$. On the other hand, $i_{q B}$ is significant affected by the perturbations, since the motor B is not instantaneously controlled. About the PC instead (column a), it can be noted that both $i_{q A}$ and $i_{q B}$ current behaviors are affected by similar overshoots. In particular, the MSC $i_{q B}$ overshoots are much stronger than the PC $i_{q B}$ ones. Thus, it can be concluded that the MSC, by effectively controlling only the master motor, forces all the perturbations effects to manifest on the slave motor. The proposed "mean motor" approach, instead, by effectively allowing the perturbations effects to be shared by both motors, guarantees that the maximum currents overshoot is smaller than the one related to MSC.

Another difference between the two methods can be highlighted by looking at the angular speed behaviors. The first overshoots at $t=3 \mathrm{~s}$ (loading of motor $\mathrm{B}$ ) is smaller for the MSC, while the second overshoots at $t=11 \mathrm{~s}$ (loading of motor A) is smaller for the PC. Indeed, as already highlighted in [1] for the MSC approach, while the master motor speed dynamic is controlled by the speed regulator, the slave motor speed dynamic is regulated by the damping controller; this last is in fact characterized by a higher bandwidth. In contrast, the PC, as already stated, has no bias for one of the two motors, and presents the same overshoots, which anyway appear smaller than those linked to the master motor of MSC. The same considerations can be applied in correspondence of the motor B unloading event $(t=14 \mathrm{~s})$ and the motor A unloading event $(t=17 \mathrm{~s})$.

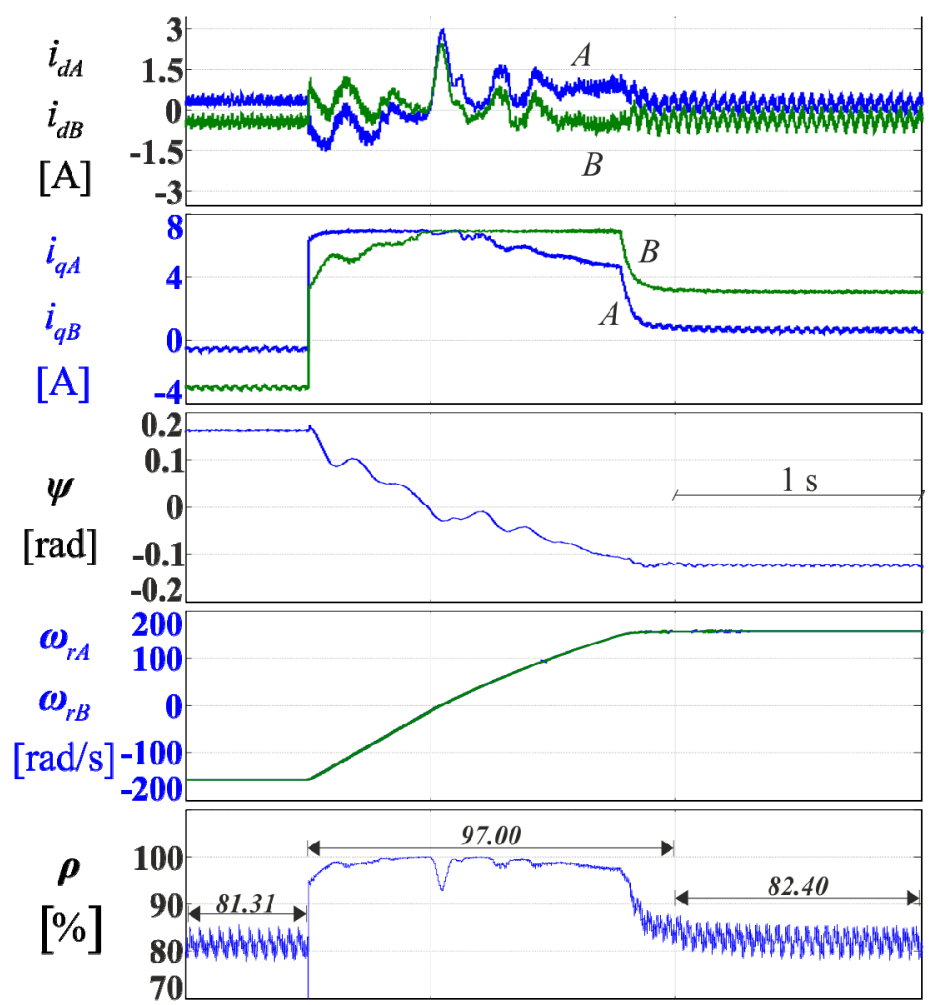

(a)

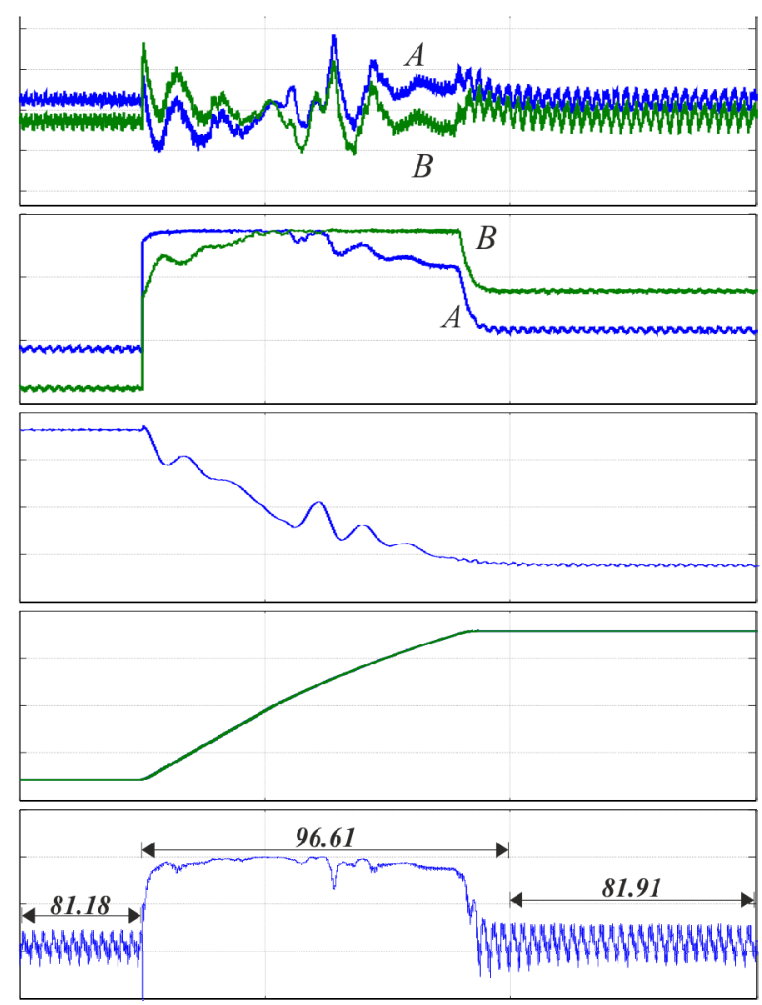

(b)

Fig. 12. Experimental results during reference speed sign inversion showing $i_{d A}, i_{d B}, i_{q A}, i_{q A}, \psi, \omega_{r A}, \omega_{r B}, \rho:$ a) PC; b) MSC. 
Finally, regarding the behavior of $\rho$, the PC guarantees smaller ripples during steady state conditions and, consequently, slightly higher mean values (reported in Fig. 11 with bold italicized numbers). Indeed, the PC, by controlling $\psi$ through a double cascaded loop, is able to limit its oscillations during steady state, as clearly noticeable in the first $3 \mathrm{~s}$ zoom of the $\psi$ behavior. During transients, as a consequence of the $\mathrm{PC}$ smoother currents behaviors, the ratio $\rho$ presents smaller overshoots for the PC; this is particularly evident at $t=14 \mathrm{~s}$ (motor B unloading).

In order to test the effectiveness of the PC across zero speed, starting from a value of $-0.5 \mathrm{pu}$ and with only motor B at the rated loaded, a speed inversion was initiated at time $0.5 \mathrm{~s}$. Fig. 12 shows the experimental results. Again, the outcomes of the two approaches appear quite similar. Few differences can be found in the mean values of $\rho$ (higher in each case for the PC), and in the behavior of $\psi$, which appears smoother for the PC, especially around the zero crossing.

Another intrinsic difference between PC and MSC is related to how the two methods approach the calculation of the optimum condition. Although the derivative terms of model (3) are neglected in both methods, so that they aim to reach an optimum solution only in steady state, the PC obtains the reference $\psi^{*}$ value based on the torque references and speed value, thus providing an available solution under both transient and steady state conditions.

In contrast, MSC faces a quartic function, which can be approached using either a numeric iterative method or closed solution, giving the optimum $d$-axis current component of the master motor $\left(i_{d, M T P A}\right)$, i.e. the steady state component of the $d$-axis reference current of the master motor. Because the quartic function, derived using steady state equations, is based on the actual current values, it has no solution in presence of strong currents derivatives; this leads to strong oscillations of $i_{d, M T P A}$ values during transients. Thus, a low pass filter is required for $i_{d, M T P A}$ while a damping controller is used to stabilize the system performance. Indeed, the behavior of $i_{d, M T P A}$ during the speed inversion test, reported in Fig. 13 (blue line) together with its filtered version (red line), is characterized by strong oscillations.

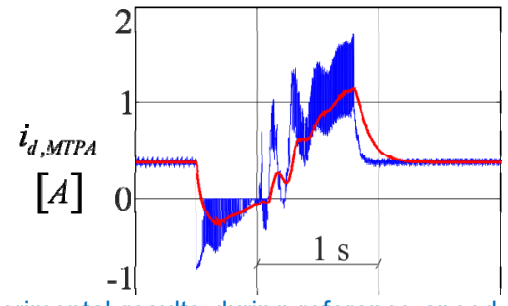

Fig. 13. Experimental results during reference speed sign inversion showing $i_{d, M T P A}$ : (blue line) and its filtered version (red line) for MSC.

In conclusion, during transient operations, while the $d$-axis current component of the master motor is substantially determined by the damping term, the MSC optimal tracking algorithm just increases the computational burden. This consideration is validated by Fig. 14, which shows the computational time of $\mathrm{PC}$ and MSC algorithms during speed inversion. It can be noted that the MSC computational time significantly increases when $i_{d, M T P A}$ oscillations arise, reaching a maximum value 1.6 greater than that related to the PC.
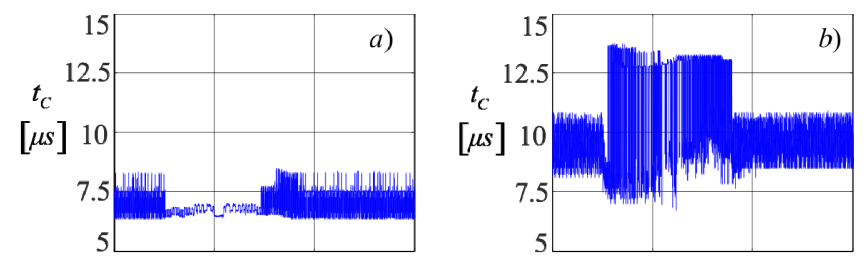

Fig. 14. Computational time tc during reference speed sign inversion for: a) PC; b) MSC.

\section{CONCLUSIONS}

With reference to a unified mathematical model for MIDP PMSM drives, this paper has considered the optimum control problem.

Two different control strategies have been considered, and the expressions of their objective functions have been derived through the introduction of new variables, linked to the load conditions, making the considerations about the optimal tracking independent of the motor parameters. Simplified closed analytical expressions for the MTPA have been derived, independently of steady state or transient conditions, and selected via a parameter independent look-up table.

An innovative control diagram has been proposed and experimentally tested on a $1400 \mathrm{~W}$ test bench, with the results compared to those of the present state of the art through a complete rated loading sequence and during the speed inversion. The parameter uncertainties have also been investigated. The proposed simplified technique, although characterized by a lower computational burden, has shown an equivalent experimental performance.

\section{APPENDIX}

By fixing the limit current $I_{L}$ and demagnetizing limit current $I_{s}$, the minimum and Maximum values of $i_{d \Sigma}$ $\left(i_{d \Sigma, L, m}, i_{d \Sigma, L, M}\right)$, which guarantee that the magnitude of the currents flowing in motors $\mathrm{A}$ and $\mathrm{B}$ do not overcome $I_{L}$, and the minimum value of $i_{d \Sigma}\left(i_{d \Sigma, S, m}\right)$, which guarantees that the direct components do not reach $I_{s}$, can be calculated at $i_{q \Sigma}=0$ via the following position:

$\left\{\begin{array}{l}I_{d \Sigma, A, L}^{\text {low }}=-i_{d \Delta}-\sqrt{I_{L}^{2}-i_{q \Delta}^{2}}, \quad I_{d \Sigma, A, L}^{\text {high }}=-i_{d \Delta}+\sqrt{I_{L}^{2}-i_{q \Delta}^{2}} \\ I_{d \Sigma, B, L}^{\text {low }}=i_{d \Delta}-\sqrt{I_{L}^{2}-i_{q \Delta}^{2}}, \quad I_{d \Sigma, B, L}^{\text {high }}=i_{d \Delta}+\sqrt{I_{L}^{2}-i_{q \Delta}^{2}} \\ I_{d \Sigma, A, S}=-\frac{1}{\cos \psi} I_{S}-i_{d \Delta}+\tan \psi i_{q \Delta} \\ I_{d \Sigma, B, S}=-\frac{1}{\cos \psi} I_{S}+i_{d \Delta}+\tan \psi i_{q \Delta}\end{array}\right.$

and correspond to the following: 


$$
\left\{\begin{array}{l}
i_{d \Sigma, L, m}=\max \left(I_{d \Sigma, A, L}^{\text {low }}, I_{d \Sigma, B, L}^{\text {low }}\right) \\
i_{d \Sigma, L, M}=\min \left(I_{d \Sigma, A, L}^{\text {high }}, I_{d \Sigma, B, L}^{\text {high }}\right) \\
i_{d \Sigma, S, m}=\max \left(I_{d \Sigma, A, S}, I_{d \Sigma, B, S}\right)
\end{array}\right.
$$

Consequently, the admissible range for $i_{d \Sigma}^{*}$ is as follows:

$i_{d \Sigma}^{*} \in\left[\max \left(i_{d \Sigma, S, m}, i_{d \Sigma, L, m}\right), i_{d \Sigma, L, M}\right]$

Once $i_{d \Sigma}^{*}$ has been determined by limiting $i_{d \Sigma, n l}^{*}$ as per (12), the equivalent quantities $i_{q \Sigma, L, m}, i_{q \Sigma, L, M}, i_{q \Sigma, S, m}$ for $i_{q \Sigma}$ can be calculated via the following position:

$$
\left\{\begin{array}{l}
I_{q \Sigma, A, L}^{\text {low }}=-i_{q \Delta}-\sqrt{I_{L}^{2}-\left(i_{d \Sigma}^{*}+i_{d \Delta}\right)^{2}} \\
I_{q \Sigma, A, L}^{\text {high }}=-i_{q \Delta}+\sqrt{I_{L}^{2}-\left(i_{d \Sigma}^{*}+i_{d \Delta}\right)^{2}} \\
I_{q \Sigma, B, L}^{\text {low }}=i_{q \Delta}-\sqrt{I_{L}^{2}-\left(i_{d \Sigma}^{*}-i_{d \Delta}\right)^{2}} \\
I_{q \Sigma, B, L}^{\text {high }}=i_{q \Delta}+\sqrt{I_{L}^{2}-\left(i_{d \Sigma}^{*}-i_{d \Delta}\right)^{2}} \\
I_{q \Sigma, A, S}=\frac{1}{\sin \psi}\left(I_{S}+\cos \psi i_{d \Sigma}^{*}\right)+\cot \psi i_{d \Delta}-i_{q \Delta} \\
I_{q \Sigma, B, S}=-\frac{1}{\sin \psi}\left(I_{S}+\cos \psi i_{d \Sigma}^{*}\right)+\cot \psi i_{d \Delta}+i_{q \Delta}
\end{array}\right.
$$

and correspond to the following:

$$
\begin{cases}i_{q \Sigma, L, m}=\max \left(I_{q \Sigma, A, L}^{l o w}, I_{q \Sigma, B, L}^{l o w}\right) & \\ i_{q \Sigma, L, M}=\min \left(I_{q \Sigma, A, L}^{\text {high }}, I_{q \Sigma, B, L}^{\text {high }}\right) & \\ i_{q \Sigma, S, m}=I_{q \Sigma, B, S}, \quad i_{q \Sigma, S, M}=I_{q \Sigma, A, S} & (\text { with: } \psi>0) \\ i_{q \Sigma, S, m}=I_{q \Sigma, A, S}, \quad i_{q \Sigma, S, M}=I_{q \Sigma, B, S} & \text { (with: } \psi<0)\end{cases}
$$

In (14), the quantity $i_{q \Sigma, S, M}$ represents the Maximum value of $i_{q \Sigma}$, which guarantees that the direct components of the currents flowing in each motor do not reach $I_{s}$. Consequently, the admissible range for $i_{q \Sigma}^{*}$ is as follows:

$i_{q \Sigma}^{*} \in\left[\max \left(i_{q \Sigma, L, m}, i_{q \Sigma, S, m}\right), \min \left(i_{q \Sigma, L, M}, i_{q \Sigma, S, M}\right)\right]$

\section{REFERENCES}

[1] Y. Lee and J. I. Ha, "Control Method for Mono Inverter Dual Paralle Surface-Mounted Permanent-Magnet Synchronous Machine Drive System," IEEE Transactions on Industrial Electronics, vol. 62, no. 10, pp. 6096-6107, Oct. 2015.

[2] R. Pen-Eguiluz, M. Pietrzak-David, V. Riga and B. de Fornel, "Comparison of several speed sensorless strategies of two different dual drive induction motor control structures," VIII IEEE International Power Electronics Congress, 2002. Technical Proceedings. CIEP 2002, pp. 41 46.

[3] D. Yıldırım and B. Caglar, "A new control technique for improving dynamic performance of mono inverter dual parallel induction motors in railway traction systems," 2015 Intl Symposium on Advanced Electromechanical Motion Systems (ELECTROMOTION), pp. 756-761.

[4] F. Xu, L. Shi, and Y. Li, "The weighted vector control of speedirrelevant dual induction motors fed by the single inverter," IEEE Transactions on Power Electronics., vol. 28, no. 12, pp. 5665-5672, Dec. 2013.

[5] K. Matsuse, H. Kawai, Y. Kouno, and J. Oikawa, "Characteristics of speed sensorless vector controlled dual induction motor drive connected in parallel fed by a single inverter," IEEE Transactions on Industry Application, vol. 40, no. 1, pp. 153-161, Jan./Feb. 2004.

[6] A. Bouscayrol et al., "Weighted control of traction drives with parallel connected ac machines," IEEE Transactions on Industrial Electronics, vol. 53, no. 6, pp. 1799-1806, Dec. 2006.

[7] D. Bidart, M. Pietrzak-David, P. Maussion and M. Fadel, "Mono inverter dual parallel PMSM - structure and control strategy," 2008 34th Annual Conference of IEEE Industrial Electronics, Orlando, FL, 2008, pp. 268-273.

[8] D. Bidart, M. Pietrzak-David, P. Maussion, and M. Fadel, "Mono inverter multi-parallel permanent magnet synchronous motor: Structure and control strategy," IET Electric Power Applications, vol. 5, no. 3, pp. 288-294, Mar. 2011.

[9] N. L. Nguyen, M. Fadel, and A. Llor, "A new approach to predictive torque control with dual parallel PMSM system," IEEE International Conference on Industrial Technology, 2013, pp. 1806-1811.

[10] N. L. Nguyen, M. Fadel and A. Llor, "Predictive Torque Control - A solution for mono inverter-dual parallel PMSM system," 2011 IEEE International Symposium on Industrial Electronics, Gdansk, 2011, pp. 697-702.

[11] M. Fadel, N. L. Nguyen and A. Llor, "Direct Torque Control - A solution for mono inverter-dual parallel PMSM system," 21st Mediterranean Conference on Control and Automation, Chania, 2013, pp. 1477-1483.

[12] T. Liu and M. Fadel, "Comparative study of different predictive torque control strategies for mono-inverter dual-PMSM system," 2016 18th Mediterranean Electrotechnical Conference (MELECON), Lemesos, 2016, pp. 1-6.

[13] A. Del Pizzo, D. Iannuzzi and I. Spina, "High performance control technique for unbalanced operations of single-vsi dual-PM brushles motor drives," 2010 IEEE International Symposium on Industrial Electronics, Bari, 2010, pp. 1302-1307.

[14] A. Del Pizzo, D. Iannuzzi and I. Spina, "Optimum Torque/Current Control of Dual-PMSM Single-VSI Drive”, Electronics, vol. 14(1) June 2010, pp.61-66

[15] M. S. D. Acampa, A. Del Pizzo, D. Iannuzzi, I. Spina "Predictive control technique of single inverter dual motor AC-brushless drives", 2008 International Conference on Electrical Machines, Vilamoura, 2008, pp. $1-6$.

[16] J. M. Lazi, Z. Ibrahim,M. H. N. Talib, and R. Mustafa, "Dual motor drives for PMSM using average phase current technique," IEEE International Conference on Power and Energy, 2010, pp. 786-790.

[17] Yongjae Lee and J. I. Ha, "Minimization of stator currents for mono inverter dual parallel PMSM drive system," International Power Electronics Conference (IPEC-Hiroshima - ECCE ASIA), Hiroshima, 2014, pp. 3140-3144.

[18] Y. Lee and J. I. Ha, "Control method for mono inverter dual parallel interior permanent magnet synchronous machine drive system," 2015 IEEE Energy Conversion Congress and Exposition (ECCE), Montreal, QC, 2015, pp. 5256-5262.

[19] Y. Lee and J. I. Ha, "Analysis of parameter variations on mono inverter dual parallel SPMSM drive system," 2015 9th International Conference on Power Electronics and ECCE Asia (ICPE-ECCE Asia), Seoul, 2015, pp. 1875-1880.

[20] Y. Lee and J. I. Ha, "Analysis and control of mono inverter dual parallel SPMSM drive system," 2014 IEEE Energy Conversion Congress and Exposition (ECCE), Pittsburgh, PA, 2014, pp. 4843-4849.

[21] Y. Kim and J. I. Ha, "Novel topology and control of single inverter system for two permanent magnet synchronous machines," 2014 IEEE Applied Power Electronics Conference and Exposition - APEC 2014, Fort Worth, TX, 2014, pp. 833-837.

[22] Lee, Y., Ha, J.-I. "Control method for mono inverter dual parallel interior permanent magnet synchronous machine drive system" (2015) 2015 IEEE Energy Conversion Congress and Exposition, ECCE 2015, art. no. 7310399 , pp. 5256-5262. 